\title{
Oromucosal Cream Dosage Form
}

National Cancer Institute

\section{Source}

National Cancer Institute. Oromucosal Cream Dosage Form. NCI Thesaurus. Code C149733.

Semi-solid, usually multidose preparation consisting of an oil-in-water emulsion intended for oromucosal use. Oromucosal creams are applied to the oral cavity or onto a specific part of the oral cavity, other than the gingivae, to obtain a local effect. 